\title{
DOSE VOLUME EFFECT OF ACUTE DIARRHEA IN POST-OPERATIVE RADIATION FOR GYNECOLOGIC CANCER
}

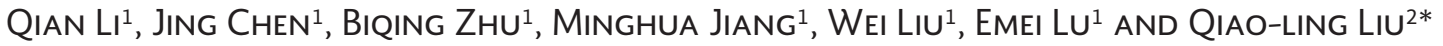 \\ ${ }^{1}$ Department of Radiation Oncology, The Affiliated Cancer Hospital of Nanjing Medical University, Jiangsu Cancer \\ Hospital, Jiangsu Sheng; ${ }^{2}$ Department of Obstetrics and Gynecology, Nanjing Jiangning Hospital, The Affiliated Jiangning \\ Hospital of Nanjing Medical University, Nanjing, China
}

\begin{abstract}
Background: Diarrhea is the primary symptom of concern in acute post-operative radiation-induced enteritis in gynecologic cancer. We retrospectively studied the correlation between the volume of irradiated small bowel and the development of acute diarrhea in these patients. Materials and Methods: A total of 100 post-operative gynecologic cancer patients were analyzed. Pelvic computed tomography was performed to calculate the volume of irradiated small bowel. A dose-volume histogram was calculated from 5 to $40 \mathrm{~Gy}$ at $5 \mathrm{~Gy}$ intervals. Patients receiving conventional whole pelvic radiation therapy (RT) were assigned to Group I, and those who received intensity-modulated RT (IMRT) were assigned to Group II. A total dose of 40-50 Gy was delivered at 1.8-2.0 Gy per fraction daily. Acute diarrhea during treatment was scored. All data were expressed as a mean \pm standard deviation. Different dose-volume parameters for small bowel in Grades $0-1$ and Grades 2-3 diarrhea were calculated by the independent $t$-test. Univariate analysis of diarrhea risk factors was performed with the independent $t$-test or Chi-square/Fisher exact test. Results: Of the 77 patients who received conventional RT, 44 (57.14\%) experienced Grades 2-3 toxicities. Of the 23 patients who received IMRT, 9 (39.13\%) experienced Grades 2-3 toxicities. Concurrent chemotherapy was slightly associated with a higher damage score in both groups $(p=0.028)$. None of the patient factors (weight, percentage depth dosage, dose fraction, distance from skin to tumor, lymph node metastasis, chemotherapy, block, brachytherapy, hypertension, or diabetes) were correlated with diarrhea in the two groups. The volumes of irradiated small bowel in patients who experienced Grades 2-3 diarrhea were significantly larger than those in patients who experienced Grades 0-1 diarrhea at all dose levels in Group I. V20 (372.19 $\left.\pm 133.26 \mathrm{~cm}^{3}, \mathrm{p}=0.004\right)$ was an independent factor for developing Grades 2-3 diarrhea in Group I. V25 (290.35 $\pm 130.22 \mathrm{~cm}^{3}, \mathrm{p}=0.001$ ) was an independent risk factor for all patients who developed higher score diarrhea. Conclusions: The volume of irradiated small bowel was an independent risk factor for all patients who developed diarrhea, especially those undergoing conventional RT.
\end{abstract}

Key words: Acute diarrhea. Conventional radiotherapy. Gynecologic cancer. Intensity-modulated radiation therapy.

\author{
Corresponding author: \\ *Qiao-Ling Liu \\ Department of Obstetrics and Gynecology \\ Nanjing Jiangning Hospital \\ The Affiliated Jiangning Hospital of \\ Nanjing Medical University \\ Gushan Road 168 \\ Jiangning District, Nanjing, China \\ E-mail: liuqlnjh@126.com
}

Received for publication: 16-08-2017

Approved for publication: 03-11-2017

doi: $10.24875 /$ RIC.17002373 


\section{INTRODUCTION}

Common treatment in gynecologic cancer entails surgery, radiation, and chemotherapy. A major repercussion of these treatments is that unavoidable treatment of closely related tissues causes patients to suffer additional pain and depression. These side effects can interfere with treatment and, at times, even lead to complete treatment failure. Therefore, clinicians have been paying more attention to the critical outcome of the patient's quality of life. The criteria for assessing treatment toxicity are diverse. For radiotherapy, treatment toxicities are divided into acute and late categories. During post-operative radiation, a large proportion of small bowel that is in close proximity to the target fields is often irradiated with a substantial dose. Many reports have claimed a strong association between the volume of irradiated small bowel and the development of enteritis complications ${ }^{1,2}$. The primary symptom of concern in acute radiation-induced enteritis is diarrhea ${ }^{3}$. Although other symptoms, such as nausea and abdominal pain, are more common, the severity of these symptoms is affected by the patients' tolerance and the difficulty with their precise measurement.

Post-operative radiation therapy (RT) is used for patients with a high risk of tumor micrometastasis after surgery ${ }^{4}$. The normal tissues, such as the small and large bowels, settle into the pelvic resection cavities after surgery, which often encompass the target volume. If that is the case, the entirety of pelvic tissue that is located in the high-dose volume could contribute to radiation-related injury. Intensity-modulated RT (IMRT) programs precisely conform to the isodose lines in the shape of the target volume with the intention of protecting the normal tissue from an unnecessarily high radiation dose ${ }^{5}$. Conventional RT technique is often used in large cancer centers due to the high demand of resources and time of the IMRT. The target fields of radiotherapy include the upper half of the vagina, uterus, parauterine and pelvic lymph nodes $(L N)$, and even $L N$ of the abdominal aorta. Therefore, the irradiation target field for cervical cancer is rather irregular, involving considerable volumes of the small bowel, rectum, and bladder. The tolerances of pelvic organs to radiotherapy are obviously different, with the small bowel being the most sensitive (minimum tolerance dose TD5/5: $50 \mathrm{~Gy}$ ). The tolerance of the bladder and rectum is $60 \mathrm{~Gy}$, while that of the uterus and upper half of the vagina is high (over $90 \mathrm{~Gy}$ ). Thus, it is imperative to overcome gastrointestinal and urinary complications induced by pelvic radiotherapy for malignant gynecologic tumors. The incidence rates of severe complications after pelvic radiotherapy at 45-50 Gy can reach $4-15 \%$. Over $40 \%$ of patients suffer from long-term chronic diarrhea, and $2 \%-8 \%$ of cases undergo serious bladder complications. Since the pelvis is also irradiated, the hematologic system may also be subjected to complications. The dose of routine irradiation for $L N$ of the abdominal aorta is limited by surrounding involved organs such as the small bowel, kidney, and spinal cord, mostly below 40-50 Gy ${ }^{6,7}$. Until now, the relationship between the dose of radiotherapy for gynecologic cancer and acute diarrhea has seldom been reported.

With this motivation, we retrospectively studied the characteristics and dosimetric factors of patients who had received conventional RT or IMRT for gynecologic malignancy. The purpose of the study was to investigate the difference in toxicities between conventional RT and IMRT and to analyze the dose-volume effect for acute diarrhea with these RT techniques to improve our understanding of the threshold point, which would require extra attention.

\section{MATERIALS AND METHODS}

\section{Patient characteristics}

A total of 100 patients treated at the Affiliated Cancer Hospital of Nanjing Medical University between January 2013 and June 2014 were selected. This study was approved by the Ethical Committee of our hospital. All patients and their families had been informed about the objective and significance of this study, and they all signed consents. Cervical cancer cases included squamous-cell carcinoma, adenocarcinoma and adenosquamous carcinoma. Patients with endometrial cancer were treated only with RT. Inclusion criteria: Patients who were diagnosed as having cervical cancer or endometrial cancer; patients who were compliant with treatment throughout this study. Exclusion criteria: Patients who had intestinal inflammation complicated with severe diarrhea. There were 18 patients who were older than 60 years, and the other 84 patients were $\leq 60$ years old. Patients with endometrial cancer included 3 cases of FIGO Stage I, 
24 cases of Stage II, and 2 cases of Stage IV. Patients with cervical cancer included 10 cases of FIGO Stage I, 51 cases of Stage II, and 10 cases of Stage IV.

All patients initially underwent physical examination, pathological assessment, chest $\mathrm{X}$-ray, pelvic computed tomography (CT), and laboratory blood work. They were proposed to receive standard daily radiation treatment at a total dose of 40-50 Gy in five treatments per week at 1.8-2.0 Gy per fraction. Patient demographic information is listed in table 1 . Patients who received conventional whole pelvic RT (anterior-posterior/posterior-anterior [AP/PA] fields) were assigned to Group I $(n=77)$, and those who received IMRT were assigned to Group II $(n=23)$. Clinical variables, including age, weight, LN metastasis, chemotherapy, dose fraction, distance from source to tumor, percentage depth dosage, block, brachytherapy,

Table 1. Patient characteristics.

\begin{tabular}{|c|c|c|}
\hline Characteristic & $\begin{array}{l}\text { Number of } \\
\text { patients }(\%)\end{array}$ & Mean \pm SD \\
\hline Age & & $48 \pm 10.34$ \\
\hline$<60$ & $84(84)$ & \\
\hline$\geq 60$ & $16(16)$ & \\
\hline Group I: conventional RT & $77(77)$ & \\
\hline Group II: IMRT & $23(23)$ & \\
\hline \multicolumn{3}{|l|}{ Histological feature } \\
\hline Squamous carcinoma & $64(64)$ & \\
\hline Adenocarcinoma & $29(29)$ & \\
\hline Others & $7(7)$ & \\
\hline Cervix carcinoma & $71(71)$ & \\
\hline Endometrial carcinoma & $29(29)$ & \\
\hline \multicolumn{3}{|l|}{ FIGO stage of endometrial cancer } \\
\hline I & $3(10.3)$ & \\
\hline$\|$ & $24(82.8)$ & \\
\hline III & 0 & \\
\hline IV & $2(6.9)$ & \\
\hline \multicolumn{3}{|l|}{ FIGO stage of cervical cancer } \\
\hline I & $10(14.1)$ & \\
\hline II & $51(71.8)$ & \\
\hline III & 0 & \\
\hline IV & $10(14.1)$ & \\
\hline \multicolumn{3}{|l|}{ Pelvic LN metastasis } \\
\hline \multicolumn{3}{|l|}{ Endometrial cancer (29) } \\
\hline Negative & $17(58.6)$ & \\
\hline Positive & $3(17.2)$ & \\
\hline No data or surgical records lost & $9(31.0)$ & \\
\hline \multicolumn{3}{|l|}{ Cervical cancer (71) } \\
\hline Negative & $48(67.6)$ & \\
\hline Positive & $14(16.9)$ & \\
\hline No data or surgical records lost & $9(12.7)$ & \\
\hline Diabetes & $3(3)$ & \\
\hline Hypertension & $12(12)$ & \\
\hline
\end{tabular}

RT: radiation therapy; IMRT: intensity-modulated radiation therapy; LN: lymph nodes hypertension, diabetes, and volumes, were assessed independently by two experienced physicians.

\section{Treatment plans}

Seventy-seven patients in Group I received conventional RT using 15-MV photon beams with the exception of one patient who was below the threshold body weight for 15-MV photon beams and received 6-MV beams instead. Treatment was delivered within the whole pelvic field (AP/PA fields) extending from the bottom of obturator foramina to the L4-5 interspace or superior aspect of $L 4$. The lateral border was set at $1.5 \mathrm{~cm}$ lateral to the widest diameter of the pelvic inlet. Corner blocks were used in the treatment plans of 55 patients.

Patients were immobilized on a vacuum bag cradle in supine position. The clinical target volume was contoured in accordance with previous consensus recommendations for all patients and included the upper segment of the vagina, parametrial tissues and LN regions (common, external, internal iliac, and presacral $\mathrm{LN}$ regions) ${ }^{8}$, and a $7-\mathrm{mm}$ margin around the vessels was maintained, excluding bone or muscle. Inverse plans were operated on Varian Eclipse (platform 10.0).

Concurrent chemotherapy was delivered every 3 weeks and consisted of paclitaxel that was administered at a total dose of $180 \mathrm{mg}$ intravenously over $3 \mathrm{~h}$ on day 1 , and nedaplatin that was administered at a maximum dosage of $100 \mathrm{mg}$ on day 1 or $30 \mathrm{mg}$ on day 1,2 , and 3 .

\section{Normal tissue assessment}

All the 100 enrolled patients completed this study, without loss of follow-up. Acute toxicity was scored retrospectively using the common terminology criteria for adverse events version 4.0. Grade 1 is a mild symptom with an increase of $<4$ stools per day; Grade 2 is a moderate symptom with an increase of 4-6 stools per day; Grade 3 is a severe symptom with an increase of $\geq 7$ stools per day; and Grades 4 and 5 are life-threatening and death, respectively, which did not occur in this series. Four patients withdrew from the study due to severe pain from hemorrhoids. Among those 4 patients, two of them had received a dose of $<30 \mathrm{~Gy}$, and the other 2 received more than $30 \mathrm{~Gy}$. 
Before the examination, all patients were subjected to $\mathrm{CT}$ scan to contour possibly involved organs. Both opacified and unopacified small bowel loops were contoured on each CT slice, and every dose-volume histogram was calculated for each patient from $5 \mathrm{~Gy}$ to 40 Gy at 5 Gy dose level intervals. 20 patients did not have $\mathrm{CT}$ examinations at our cancer center; therefore, the absolute volume parameters were recorded.

The diagnostic criterion for $L N$ metastasis was that CT disclosed that short-axis diameters of $\mathrm{LNs}$ were $\geq$ $1.0 \mathrm{~cm}$. Staging was performed according to the staging criteria for gynecologic cancers.

Data were obtained from both the patient medical records during treatments and the monthly follow-up telephone questionnaire through May 2016.

\section{Statistical analysis}

All data were expressed as the mean \pm standard deviation. Different dose-volume parameters for small bowel in Grades 0-1 and Grades 2-3 diarrhea were calculated by independent $t$-test. Univariate analysis of diarrhea risk factors was calculated with the independent $t$-test or Chi-square/Fisher exact test. Logistic multivariate analyses of Grades 2-3 diarrhea in Groups I and II were performed. The statistics was performed using SPSS 11.0. $p<0.05$ was considered to be statistically significant. $p$-values were obtained from a two-sided test.

\section{RESULTS}

\section{Comparison of characteristics between Groups I and II}

Patient demographic information, treatment profile and disease profile are displayed in table 2. No significant difference between groups was found for any of these factors except for age and weight. As shown in table $1 \mathrm{~S}$, the dose-volume parameters of small bowel in the two groups differed significantly from V20 to V40 ( $p<0.05)$. V20 of irradiated small bowel was $326.82 \pm 136.42 \mathrm{~cm}^{3}$ in Group I while V20 of irradiated small bowel was $253.35 \pm 75.55 \mathrm{~cm}^{3}$ in Group II patients. When we compared the V40 in these two groups, the small-bowel V40 was $81.9 \pm 41.37 \mathrm{~cm}^{3}$ in Group II patients, which was nearly one-third of the value for Group I (293.58 $\left.\pm 131 \mathrm{~cm}^{3} ; \mathrm{p}<0.001\right)$.

Of the 77 patients who received conventional RT, 44 (57.14\%) experienced Grades 2-3 toxicities. Of the 23 patients who received IMRT, 9 (39.13\%) experienced Grades 2-3 toxicities. No patients developed acute toxicity Grades 4 or 5. In Group I, 4 patients developed intestinal obstruction during follow-up between 3 months and 1 year and proceeded directly to surgery. No patients developed an intestinal obstruction in Group II as of May 2016. A total of 10 patients were given an RT break due to severe diarrhea for $<3$ days in Group I. The relationship between acute diarrhea and the small bowel volume is illustrated in tables $2 \mathrm{~S}-4 \mathrm{~S}$.

\section{Correlation between groups characteristics and acute toxicity}

None of the patient factors (weight, percentage depth dosage, dose fraction, distance from skin to tumor, LN metastasis, chemotherapy, block, brachytherapy, hypertension, or diabetes) correlated with diarrhea in the two groups. The one exception was that patients experiencing Grades 2-3 diarrhea were older than those with Grades 0-1 diarrhea in Group II $(p<0.001)$. Concurrent chemotherapy tended to associate with a higher damage score in both groups $(9.09 \%$ vs. $18.18 \%$ in Group I, and $14.29 \%$ vs. $22.22 \%$ in Group II), although the difference did not reach statistical significance (Tables $2 \mathrm{~S}$ and $3 \mathrm{~S}$ ).

A statistically significant association was observed between Grades 0-1 and Grades 2-3 diarrhea at every dose threshold level from 5 Gy to $40 \mathrm{~Gy}$ in Group I (V5: $291.77 \pm 118.32 \mathrm{~cm}^{3}$ vs. $406.94 \pm 137.46 \mathrm{~cm}^{3}$, $\mathrm{p}=0.002 ; \mathrm{V} 40: 226.49 \pm 105.57 \mathrm{~cm}^{3}$ vs. $336.44 \pm$ $128.79 \mathrm{~cm}^{3}, \mathrm{p}=0.001$ ) (Table 2S). In Group I, patients who experienced Grades 2-3 diarrhea had a significantly greater irradiated small bowel volume than patients who experienced Grades 0-1 diarrhea at every dose threshold level. In Group II, the volumes of irradiated small bowel in patients with Grades 2-3 toxicity were slightly greater than those with Grades 0-1 toxicity, but the difference was not statistically significant (Table 3S).

We then conducted unconditional multivariate logistic regression analysis of the data. We included factors 
Table 2. Comparison of characteristics between Groups I and II.

\begin{tabular}{|c|c|c|c|}
\hline Characteristic & Group I & Group II & $\mathrm{p}$ \\
\hline Age & $49.68 \pm 10.02$ & $42.39 \pm 9.57$ & 0.003 \\
\hline Age classification (\%) & & & 0.109 \\
\hline$<60$ & $62(80.50)$ & $22(95.70)$ & \\
\hline$\geq 60$ & $15(19.50)$ & $1(4.30)$ & \\
\hline Weight (kg) & $60.61 \pm 8.42$ & $54.5 \pm 5.97$ & 0.002 \\
\hline Dose fraction (Gy) & $1.85 \pm 0.04$ & $1.98 \pm 0.07$ & 0.000 \\
\hline Distance from source to tumor $(\mathrm{cm})$ & $109.78 \pm 0.96$ & $100 \pm 0$ & 0.000 \\
\hline \multicolumn{4}{|l|}{ Tumor types (No.) } \\
\hline Cervix carcinoma & 53 & 18 & \\
\hline Endometrial carcinoma & 24 & 5 & \\
\hline Pelvic LN metastasis (\%) & $17(21.70)$ & $4(18.20)$ & 1.000 \\
\hline Prior chemotherapy (\%) & $34(44.20)$ & $13(56.50)$ & 0.297 \\
\hline Concurrent-chemotherapy (\%) & $11(14.30)$ & $4(17.40)$ & 0.743 \\
\hline Total chemotherapy (\%) & $38(49.40)$ & $14(60.90)$ & 0.332 \\
\hline Block (\%) & $55(71.40)$ & $0(0.00)$ & - \\
\hline Brachytherapy (\%) & $11(14.30)$ & $0(0.00)$ & - \\
\hline Hypertension (\%) & $12(16.00)$ & $0(0.00)$ & 0.063 \\
\hline Diabetes (\%) & $3(3.90)$ & $0(0.00)$ & 1.000 \\
\hline
\end{tabular}

LN: lymph nodes

such as age, weight, LN metastasis, chemotherapy, dose fraction, distance from source to tumor, percentage depth dosage, block, brachytherapy, hypertension, diabetes, and volumes at the different dose levels in an univariate analysis of moderate and severe diarrhea. V20 (372.19 $\left.\pm 133.26 \mathrm{~cm}^{3}, p=0.004\right)$ was the single independent risk factor for Grades 2-3 diarrhea in Group I (Table 4S). V25 (290.35 $\pm 130.22 \mathrm{~cm}^{3}$, $p=0.001$ ) was an independent risk factor for Grades 2-3 diarrhea when the two groups were pooled for analysis.

\section{DISCUSSION}

Diarrhea is a manifestation of both acute and late intestinal toxicity from radiation treatment. Intestinal mucosa cells are especially vulnerable to short bursts of radiation because their high cell turnover during active phases of mitosis leads to absorption reduction and secretory disability, ultimately resulting in ulceration and fibrosis of the intestine 9 . Previous studies have reported diarrhea prevalence of approximately $30-40 \%$ in patients undergoing radiotherapy ${ }^{10}$ and have been observed to be as high as $52 \%^{11}$. Chen et al. reported that $25-35 \%$ of patients receiving chemoradiation had developed score $\geq 3$ diarrhea ${ }^{12}$. When patient self-reports were used to assess symptoms, 66\% had developed score 2 diarrhea, which was a higher incidence than that reported by physician assessments (approximately 30\%). During our follow-ups, up to $53 \%$ of all patients developed acute Grades 2-3 diarrhea, meaning that more than half of the patients suffered from acute diarrhea during cancer treatment. These data suggest that clinicians must take the high incidence of diarrhea into account during patient evaluations.

During the follow-ups, until May 2016, 7 patients in Groups I and 2 patients in Group II had chronic diarrhea. Due to the limited number of chronic events, no statistics was performed in late intestinal toxicity, and only acute bowel toxicity was included. However, emerging evidence has demonstrated an apparent relationship between acute and late radiation-induced intestinal injury, specifically in the case of small bowel damage. Previous research found that the occurrence of severe acute bowel damage significantly increased the risk of late toxicity and that patients who were given a treatment break due to diarrhea also showed an increased incidence of severe late toxicity ${ }^{4}$. Other studies have also demonstrated an association between increased risk of late complication with acute injury due to pelvic radiation ${ }^{13,14}$. Given the correlation between acute and late toxicity shown in these studies, it would appear that an average dose lower than $45 \mathrm{~Gy}$ would reduce the risk of development of late injury ${ }^{15}$. Patients with the post-operative RT received an average pelvic dose $<45 \mathrm{~Gy}$ in our cancer center, which indicates that fewer late events were observed. Two cases within the RT group suffered from post-operative pelvic recurrence, whereas there was no recurrence in the IMRT group. 
Besides, our study has confirmed that the volume effect on the small bowel was strongly associated with acute diarrhea at all dose levels in conventional whole pelvic RT and that this association became marginal in the IMRT group. The multivariate analysis demonstrated that V20 $>372.19 \mathrm{~cm}^{3}$ was a single independent risk factor for Grades 2-3 diarrhea in conventional RT, which reminds clinicians to constrain V20 $372.19 \mathrm{~cm}^{3}$ by blocks or prone position. V25 > $290.35 \mathrm{~cm}^{3}$ was an independent risk factor for Grades 2-3 diarrhea when data from all patients were pooled together, which is another reference to dose-volume limitations for clinicians. Consistent with the previous reports ${ }^{2,16-18}$, lower dose irradiation seemed to play a major role in acute diarrhea toxicity, where V15 was deemed an important dose-volume threshold level for overall gastrointestinal symptoms ${ }^{2,9,16,18}$.

Previous work has demonstrated that higher radiation toxicity is associated with prior abdominal or pelvic surgery, concurrent chemotherapy, weight, older age, previous intestinal disorder, and dose $>45 \mathrm{~Gy}^{15}$. In the current study, patients in Group II were a younger. However, the age factor in multivariate analyses of toxicities (Table 4S) did not reach significant difference in two groups; other factors (weight, LN metastasis, chemotherapy, dose fraction, distance from source to tumor, percentage depth dosage, block, brachytherapy, hypertension, and diabetes) did not significantly increase the incidence of severe toxicity, largely because the average dose was < 45 Gy in post-operative patients.

In previous studies, although the combined toxicity of conventional pelvic RT with concurrent chemotherapy was acceptable, the additional toxicity due to the introduction of chemotherapy remained a notable concern. This additional toxicity usually impeded escalation of the radiation dose. The current dosage of chemotherapy used at our cancer center is lower than that reported in previous studies. This is because the standard dosage caused severe overall toxicities, especially bone marrow depression, during past treatment observations, which often resulted in interruption and discontinuation of RT treatment. In our study, the concurrent chemotherapy was marginally associated with higher damage scores in both groups. However, the difference did not reach statistical significance. Therefore, it is recommended that at a decreased dose, chemotherapy can be used as a supplementary treatment.
In this study, only patients who received whole pelvic radiation (AP/PA fields) were included instead of those with standard four-field RT technique, because we observed a high risk of osteonecrosis of the femoral head and low control of para-aortic LN tumor metastasis. Besides, most patients arriving at our center for treatments had a tumor size larger than $4 \mathrm{~cm}$, which put patients in a high risk of recurrence. A few of the patients who received four-field RT were excluded to lower the possibility of confounding factors in this study. The number of patients in the IMRT Group is much lower than the one in conventional RT Group because the patients enrolled in both groups were recorded at about the same time. What should not be ignored is that 4 patients in the conventional RT group developed intestinal obstruction and underwent surgery, partly due to the combination of AP/PA fields RT technique with transabdominal surgery. No intestinal obstruction event was observed in the IMRT group possibly due to the fewer patients in this group.

Radiation oncologists advocate the advantage of the IMRT technique, which enables the precise design of target volumes and steep dose gradients, thus reducing the volumes of organs at risk irradiated. However, most secondary tumors occur near the normal tissue irradiated at a low or intermediate dose. IMRT can decrease the small bowel dose by $>65 \%$ at the expense of increasing the volume irradiated for dose levels $<65 \%^{18,19}$.

In summary, V20 and V25 provided better diarrhea assessment values for all selected patients, especially those undergoing conventional RT. IMRT delivered a high radiation dose to a target volume that required meticulous field techniques, which may sacrifice coverage of microscopic extension of the tumor. Nevertheless, IMRT had obvious dosimetric advantages while reducing the damage to normal tissues and the incidence of complications. More studies on IMRT with larger sample sizes are ongoing in our group.

\section{Supplementary data}

Supplementary data are available at Revista de Investigación Clínica online (www.clinicalandtranslationalinvestigation.com). These data are provided by the corresponding author and published online for the benefit of the reader. The contents of supplementary data are the sole responsibility of the authors. 


\section{REFERENCES}

1. Webb GJ, Brooke R, De Silva AN. Chronic radiation enteritis and malnutrition. J Dig Dis. 2013;14:350-7.

2. Chen RC, Mamon HJ, Ancukiewicz M, et al. Dose-volume effects on patient-reported acute gastrointestinal symptoms during chemo radiation therapy for rectal cancer. Int J Radiat Oncol Biol Phys. 2012;83:e513-7.

3. Vidal-Casariego A, Calleja-Fernández A, de Urbina-González JJ, et al. Efficacy of glutamine in the prevention of acute radiation enteritis: A randomized controlled trial. JPEN J Parenter Enteral Nutr. 2014;38:205-13.

4. Huscher A, Bignardi M, Magri E, et al. Determinants of small bowel toxicity in postoperative pelvic irradiation for gynecological malignancies. Anticancer Res. 2009;29:4821-6.

5. Marta GN, Silva V, de Andrade Carvalho H, et al. Intensitymodulated radiation therapy for head and neck cancer: Systematic review and meta-analysis. Radiother Oncol. 2014; 110:9-15.

6. Schefter T, Winter K, Kwon JS, et al. RTOG 0417: Efficacy of bevacizumab in combination with definitive radiation therapy and cisplatin chemotherapy in untreated patients with locally advanced cervical carcinoma. Int J Radiat Oncol Biol Phys. 2014;88:101-5.

7. Bernard S, Ouellet MP, Moffet H, Roy JS, Dumoulin C. Effects of radiation therapy on the structure and function of the pelvic floor muscles of patients with cancer in the pelvic area: A systematic review. J Cancer Surviv. 2016;10: 351-62.

8. Small W Jr., Mell LK, Anderson P, et al. Consensus guidelines for delineation of clinical target volume for intensity-modulated pelvic radiotherapy in postoperative treatment of endometrial and cervical cancer. Int J Radiat Oncol Biol Phys. 2008; 71:428-34.

9. Hauer-Jensen M, Denham JW, Andreyev HJ. Radiation enteropathy - Pathogenesis, treatment and prevention. Nat Rev Gastroenterol Hepatol. 2014;11:470-9.
10. Fuccio L, Guido A, Andreyev HJ. Management of intestinal complications in patients with pelvic radiation disease. Clin Gastroenterol Hepatol. 2012;10:1326-340000.

11. Amzallag-Bellenger E, Oudjit A, Ruiz A, et al. Effectiveness of MR enterography for the assessment of small-bowel diseases beyond crohn disease. Radiographics 2012;32:1423-44.

12. Chen RC, Mamon HJ, Chen $\mathrm{YH}$, et al. Patient-reported acute gastrointestinal symptoms during concurrent chemo radiation treatment for rectal cancer. Cancer. 2010;116:1879-86.

13. Jhingran A, Winter $K$, Portelance $L$, et al. A phase II study of intensity modulated radiation therapy to the pelvis for postoperative patients with endometrial carcinoma: Radiation therapy oncology group trial 0418. Int J Radiat Oncol Biol Phys. 2012;84:e23-8.

14. Chopra S, Dora T, Chinnachamy AN, et al. Predictors of grade 3 or higher late bowel toxicity in patients undergoing pelvic radiation for cervical cancer: Results from a prospective study. Int J Radiat Oncol Biol Phys. 2014;88:630-5

15. Mohindra P, Bentzen SM, Giacomelli I, et al. Adjuvant Whole-Pelvic Radiation Therapy (WPRT) for endometrioid adenocarcinoma (EA): 45 Gy or 50/50.4 Gy? Int J Radiat Oncol Biol Phys. 2016;96:E300.

16. Banerjee R, Chakraborty S, Nygren I, Sinha R. Small bowel dose parameters predicting grade $\geq 3$ acute toxicity in rectal cancer patients treated with neoadjuvant chemoradiation: An independent validation study comparing peritoneal space versus small bowel loop contouring techniques. Int J Radiat Oncol Biol Phys. 2013;85:1225-31.

17. Samuelian JM, Callister MD, Ashman JB, et al. Reduced acute bowel toxicity in patients treated with intensity-modulated radiotherapy for rectal cancer. Int J Radiat Oncol Biol Phys. 2012;82:1981-7.

18. Barillot I, Tavernier E, Peignaux K, et al. Impact of post-operative intensity modulated radiotherapy on acute gastro-intestinal toxicity for patients with endometrial cancer: Results of the phase II rtcmiendometre french multicentre trial. Radiother Oncol. 2014;111:138-43.

19. Shih KK, Milgrom SA, Abu-Rustum NR, et al. Postoperative pelvic intensity-modulated radiotherapy in high risk endometrial cancer. Gynecol Oncol. 2013;128:535-9. 